\title{
EDUCAR PARA PREVENIR: A IMPORTÂNCIA DA INFORMAÇÃO NO CUIDADO DO PÉ DIABÉTICO
}

\section{EDUCATING TO PREVENT: THE IMPORTANCE OF INFORMATION IN DIABETIC FOOT CARE}

PA - BRASIL

\author{
Imaikon Gomes de Lima* \\ Joelma Flórence Lobo Costa* \\ Alana Ferreira de Oliveira*** \\ José Nunes Borges Junior ${ }^{* * * *}$ \\ Amanda Soares Peixoto ${ }^{* * * * *}$ \\ Miguel Soares Pancieri ${ }^{* * * * * * *}$ \\ Cezar Augusto Muniz Caldas* \\ Carla Andrea Avelar Pires
}

\section{RESUMO}

O artigo é referente a um estudo descritivo, transversal, oriundo do projeto de extensão intitulado "Cuidado e informação: atenção multiprofissional permanente as afecções dermatológicas em pés de pacientes diabéticos", com o objetivo de identificar alterações dermatológicas nos pés e oferecer informações e recomendações ao diabético para prevenção do pé diabético.O estudo foi realizado com 212 pacientes diabéticos atendidos no ambulatório de endocrinologia e clínica médica de um Hospital Universitário, através de entrevista e o exame físico dos pés, que mostrou o baixo nível de conhecimento sobre a Síndrome do pé diabético etambém uma associação significativa entre o maior tempo de Diabetes Mellitus e presença de lesões dermatológicas $(p=0,01)$, com destaque a xerose $(55,2 \%)$, onicomicoses $(43,9 \%)$ e

\footnotetext{
* Aluno de graduação da Universidade Federal do Pará (UFPA), PA - Brasil. E-mail: Imaikon_lima@hotmail.com

** Aluna de graduação Universidade Federal do Pará (UFPA), PA - Brasil. E-mail: elmaflc@hotmail.com

*** Aluna de graduação Universidade Federal do Pará (UFPA), PA - Brasil. E-mail: alanaferreiradeoliveira@yahoo.com.br

**** Aluno de graduação da Universidade Federal do Pará (UFPA), PA - Brasil. E-mail: borgesjuniorr@yahoo.com.br

***** Aluna de graduação da Universidade Federal do Pará (UFPA), PA - Brasil. E-mail: dinha_pretty28@hotmail.com

****** Aluno de graduação da Universidade Federal do Pará (UFPA), PA - Brasil. E-mail: miguelpancieri@gmail.com

******* Professor da Universidade Federal do Pará (UFPA), PA - Brasil. E-mail: cezar_caldas@yahoo.com.br

******** Professora da Universidade Federal do Pará (UFPA), PA - Brasil. E-mail: carlaavelarpires@gmail.com
} 
fissuras $(31,1 \%)$. Este estudo reforça a necessidade de orientar e incentivar a prática do autocuidado como forma de redução dessa complicação.

Palavras-chave: diabetes mellitus; fatores de risco; pé diabético.

\section{ABSTRACT}

This article reports a descriptive, cross-sectional study originated in the extension project titled "Care and information: permanent multiprofessional attention to dermatologic feet disorders of diabetic patients". It aims at identifying skin changes in feet, providing information about diabetic foot and offering recommendations for prevention. The study was carried out with 212 diabetic patients treated at the endocrinology and medical clinic of a university hospital through interview and physical examination of the feet, which showed the patients' low level of knowledge about diabetic foot syndrome as well as a significant association between longer diagnosis of diabetes mellitus and the presence of skin lesions $(p=0,01)$ - especially xerosis $(55,2 \%)$, onychomycosis $(43,9 \%)$ and fissures $(31,1 \%)$. This study reinforces the need to instruct and encourage the practice of self-care as a way to reduce this complication.

Keywords: diabetes mellitus; risk factors; diabetic foot.

\section{Introduçáo}

O Diabetes Mellitus (DM) é uma doença crônica que ocorre a partir de uma associaçáo de distúrbios metabólicos caracterizados por hiperglicemia, sendo resultante de defeitos na secreção e/ou na ação da insulina. Atualmente, sua classificação inclui quatro classes clínicas, sendo elas: Diabetes Mellitus tipo 1 (DM1), Diabetes Mellitus tipo 2 (DM2), outros tipos específicos de Diabetes Mellitus e Diabetes Mellitus Gestacional (SBD, 2015; ADA, 2013; ALBERTI; ZIMMET, 1999).

O DM1 e DM2 são os tipos mais comuns da doença. O DM1, por exemplo, representa de 5 a 10\% dos casos de DM e decorre da destruiçáo autoimune das células beta-pancreáticas, resultando em deficiência de insulina e ocorrendo principalmente em indivíduos jovens. O DM2, por sua vez, é visto em até $95 \%$ dos casos, é caracterizado por defeitos na ação e/ou na secreção de insulina e na regulação da produção hepática de glicose, estando frequentemente associado ao envelhecimento, sobrepeso e obesidade (SBD, 2015). A hiperglicemia crônica do diabetes está associada a danos em longo prazo, como a disfunção e falha de vários órgãos, especialmente os olhos, os rins, os nervos, o coração e os vasos sanguíneos (ADA, 2013).

As complicaçóes do DM se dividem em agudas e crônicas, sendo a hiperglicemia o fator primário no desencadeamento das mesmas. As complicaçóes crônicas se dividem em microvasculares (englobando a nefropatia, retinopatia e neuropatia diabética) e macrovasculares, resultantes de alteraçóes em grandes vasos, podendo acarretar infarto agudo do miocárdio, acidente vascular encefálico e doença vascular periférica. Tais complicaçóes crônicas do DM decorrem principalmente do longo tempo de evolução da doença, do controle inadequado e de fatores genéticos associados (CECILIO, et al., 2015; TSCHIEDEL, 2014; BARBOSA; OLIVEIRA; SEARA, 2009). A doença micro e macrovascular nos diabéticos prejudica o processo de cicatrização, sendo a isquemia um importante fator contribuinte para a amputação nesses pacientes, estando presente em cerca de $90 \%$ dos casos (BOADA, 2012).

Dentre as complicaçóes crônicas do diabetes, o pé diabético apresenta grande importância. Caracteriza-se pela presença de pelo menos uma das seguintes alteraçóes que podem ocorrer nos pés do paciente portador de DM: alteraçóes neurológicas, ortopédicas, vasculares e infecciosas, sendo a amputação de membros inferiores a consequência mais temida (CAIAFA, 2011; VIEIRA-SANTOS, 
2008; PEDROSA, 2001) e permanecendo entre as complicaçóes mais graves da doença (BAKKER; APELQVIST; SCHAPER, 2012). A neuropatia é considerada o fator mais importante no desenvolvimento desta patologia. No entanto, outros fatores presentes no paciente diabético podem ser identificados e apontam para um risco aumentado de desenvolvimento do pé diabético, como úlcera prévia, deformidades nos pés, calos, alteraçôes visuais e nefropatia (LEDESMA et al., 2008).

No que diz respeito a úlcera, ocorre o aumento da suscetibilidade à infecçáo devido à perda da função de barreira natural. A função normal dos leucócitos e do sistema imunológico são afetados pelo $\mathrm{DM}$, rebaixando a resistência do hospedeiro e aumentando a carga bacteriana e fúngica na ferida, o que favorece a chance de uma infecção extensa e mais grave (ALAVI et al., 2014). Essa complicação é uma causa significativa do crescente número de internaçóes de pacientes diabéticos, chegando a ser a quarta causa de internação de pacientes idosos e diabéticos em um hospital do Norte do país (MAUES et al., 2007).

Intervençóes específicas no estilo de vida podem reduzir a incidência da doença e, quando já diagnosticada, podem prevenir complicaçóes. Diante disso, o autocuidado tem sua importância a partir do momento em que o indivíduo começa a praticar atividades em benefício próprio, na manutenção da vida, da saúde e do bem-estar. No entanto, para que se obtenha um resultado significativo preventivo no paciente diabético, faz-se necessário o conhecimento sobre a patologia, suas complicaçóes e consequências para sua qualidade de vida (SOUZA, 2008). É de extrema importância que o paciente esteja apto a detectar problemas potenciais em seu próprio pé, tomando as medidas cabíveis e buscando ajuda de um profissional de saúde (PEDROSA, 2001). Nesse sentido, o paciente deve ser estimulado a desenvolver uma postura proativa e correta em relação ao seu autocuidado (ROCHA; ZANETTI; SANTOS, 2009). Entende-se que, com açōes conjuntas entre os serviços de saúde, profissionais que nele atuam, indivíduos e famílias, é possível o desenvolvimento de estratégias para identificar precocemente fatores de risco para o pé diabético, e assim evitá-los e/ou controlá-los (CECILIO et al., 2015).

Nesse contexto, objetiva-se estabelecer um perfil epidemiológico e clínico dos pacientes atendidos pelo projeto de extensão universitária intitulado "Cuidado e informação: atenção multiprofissional permanente as afecçôes dermatológicas em pés de pacientes diabéticos", auxiliando os profissionais de saúde no conhecimento do perfil dos indivíduos atendidos no serviço, além de mostrar a importância da orientação ao autocuidado do paciente portador da DM para a prevençáo de possíveis complicaçóes e minimizar os gastos para a saúde na região.

\section{Materiais e métodos}

Trata-se de um estudo descritivo transversal, realizado no Hospital Universitário Joáo de Barros Barreto, com 212 pacientes, todos diabéticos, atendidos no ambulatório de endocrinologia e clínica médica durante o período de fevereiro de 2014 a fevereiro de 2016. A populaçáo do estudo, selecionada por conveniência, foi composta por pacientes atendidos nos referidos ambulatórios durante a execução do projeto.

A coleta de dados foi realizada com a aplicaçáo de protocolo previamente estabelecido antes da consulta médica, enquanto os pacientes aguardavam o atendimento. Estes recebiam as informaçóes sobre os objetivos do projeto e os que concordaram 
voluntariamente em participar, assinavam o Termo de Consentimento Livre e Esclarecido (TCLE). Depois do aceite, eram submetidos ao protocolo com perguntas que abordavam sobre o conhecimento e a prática de medidas preventivas para o pé diabético, a fim de identificar o nível de conhecimento prévio sobre esta afecção.

Além disso, tinham seus pés inspecionados pelos acadêmicos participantes do projeto, anteriormente instruídos e treinados por um dermatologista. No exame, era realizada uma inspeçấo detalhada de pele e unhas dos pés dos pacientes diabéticos, após a retirada completa de calçados e meias, em busca da presença de calos, fissuras, xerose, sinais de micose interdigital ou onicomicose, corte inadequado das unhas e uso de calçados impróprios, conforme sugerido pela folha de exame físico criado pela dermatologista, coordenadora da pesquisa.

Uma vez investigadas as possíveis alteraçôes nos pés dos pacientes, eram fornecidas informaçôes sobre o conceito de pé diabético e sua etiologia, além de recomendaçôes para o cuidado do membro,por meio de um folder informativo criado pelos coordenadores e pelos alunos envolvidos, com uma linguagem acessível, e que era lido e explicado para os pacientes durante a abordagem. O texto teve como base o artigo "Pé diabético: estratégias para prevençáo" (VIGO; PACE, 2005).

As alteraçóes identificadas durante o exame físico dos pés dos pacientes eram registradas em uma ficha individual e repassadas aos responsáveis pelo atendimento do paciente, para que houvesse uma continuidade do cuidado e prescrição de demais medidas consideradas necessárias.

Para a organização e análise, os dados foram agrupados em planilhas no Microsoft Office Excel (versão 2013). As variáveis de caráter categórico (qualitativas) foram expostas através de valores absolutos e porcentagem, utilizando-se dos testes de Qui-quadrado ou o de Fisher para avaliar a diferença entre os grupos, e as variáveis quantitativas foram apresentadas através de médias e desvio-padráo, tendo sido utilizado o teste t-Student, por meio do software Bioestat versão 5.3. O valor de significância considerado estatisticamente significativo foi inferior a $5 \%(\mathrm{p}<0,05)$.

O projeto de pesquisa foi aprovado pelo Comitê de Ética em Pesquisa do Instituto de Ciências da Saúde da Universidade Federal do Pará, sob no CAAE 48201115.9.0000.0018.

\section{Resultados}

Entre os 212 pacientes diabéticos que constituíram a amostra, houve predomínio

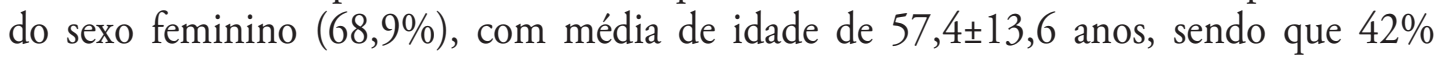
tinham idade acima dos 60 anos (Tabela 1). A maioria dos pacientes (78,3\%) atendidos nesse estudo residia na regiáo metropolitana de Belém.

Tabela 1 - Caracterização da faixa etária da populaçáo do estudo
\begin{tabular}{l|c|c|c|c}
\hline \multirow{2}{*}{ Variáveis } & \multicolumn{2}{|c}{ Sexo } & & \\
\cline { 2 - 5 } & Masculino (66) & Feminino (146) & n (212) & $\%$ \\
\hline Faixa Etária & & & & \\
\hline$<30$ & 4 & 3 & 7 & 3,3 \\
\hline 30 a 45 & 9 & 22 & 31 & 14,6 \\
\hline 46 a 60 & 27 & 58 & 85 & 40,1 \\
\hline$>60$ & 26 & 63 & 89 & 42,0 \\
\hline
\end{tabular}

Fonte: Protocolo de pesquisa 
Quanto à escolaridade, 4,7\% declaram nunca terem ido à escola e 44,3\% não concluíram o ensino fundamental (Tabela 2).

\section{Tabela 2 - Nível de escolaridade dos pacientes atendidos pela pesquisa}

\begin{tabular}{|c|c|c|}
\hline Variáveis & $\mathbf{n}$ & $\%$ \\
\hline \multicolumn{3}{|l|}{ Escolaridade } \\
\hline Analfabeto & 10 & 4,7 \\
\hline Fundamental Incompleto & 94 & 44,3 \\
\hline Fundamental Completo & 46 & 21,7 \\
\hline Ensino Médio Incompleto & 8 & 3,8 \\
\hline Ensino Médio Completo & 44 & 20,8 \\
\hline Ensino Superior Incompleto & 2 & 0,9 \\
\hline Ensino Superior Completo & 3 & 1,4 \\
\hline
\end{tabular}

A média do tempo de conhecimento da doença através do diagnóstico confirmatório

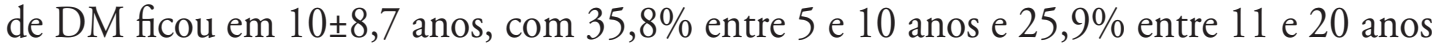
de doença diagnosticada. Em termos de tratamento do DM, foi detectado que estava sendo feito, predominantemente, apenas com antidiabéticos de uso oral, correspondendo a 58,5\% dos pacientes, ao passo que $42(19,8 \%)$ faziam uso de antidiabéticos associado à insulina, $41(19,3 \%)$ se tratavam apenas com insulina e 4 (1,9\%) estavam realizando tratamento de maneira não farmacológica.

Em relação ao conhecimento sobre a Síndrome do pé diabético, 65,6\% afirmaram não saber do que se tratava. Em contrapartida, $92(43,4 \%)$ pacientes mostraram ter alguns cuidados com seus pés (Tabela 3). Nesse contexto, notou-se que as pessoas com ensino médio e superior, geralmente, eram as que declaravam ter cuidados com os pés, como o emprego de emolientes, quando comparado àquelas que cursaram apenas o ensino fundamental ou não o concluíram $(\mathrm{p}<0,001)$.

\section{Tabela 3 - Conhecimento sobre o pé diabético e os cuidados necessários}

\begin{tabular}{l|c|c}
\hline Variáveis & $\mathbf{n}$ & \% \\
\hline Orientação sobre a doença & & \\
\hline Orientado previamente & 73 & 34,4 \\
\hline Não orientado previamente & 139 & 65,6 \\
\hline Orientação sobre a doença x cuidado com os pés & & \\
\hline Orientação prévia e cuidados especiais & 49 & 23,1 \\
\hline Orientação prévia sem cuidados especiais & 23 & 10,8 \\
\hline Sem orientação prévia com cuidados especiais & 43 & 20,3 \\
\hline Sem orientação prévia sem cuidados especiais & 96 & 45,3 \\
\hline
\end{tabular}

Fonte: Protocolo de pesquisa

Os resultados dos exames dermatológicos mostraram que a maioria dos pacientes apresentava lesóes cutâneas, resultando em uma média de, aproximadamente, 2 lesões por pessoa examinada. Entre as manifestaçóes cutâneas, destacaram-se a xerose (55,2\%), as onicomicoses $(43,9 \%)$ e as fissuras $(31,1 \%)$, ao passo que $9 \%$ dos pacientes não apresentaram nenhuma lesão (Tabela 4). 


\section{Tabela 4 - Afecçóes dermatológicas identificadas nos pés da populaçáo do estudo}

\begin{tabular}{l|c|c}
\hline Variáveis & n & \% \\
\hline Manifestaçóes cutâneas & & \\
\hline Xerose & 117 & 55,2 \\
\hline Onicomicose & 93 & 43,9 \\
\hline Fissuras & 66 & 31,1 \\
\hline Calos & 32 & 15,1 \\
\hline Úlcera Plantar & 10 & 4,7 \\
\hline Tínea Pedis & 10 & 4,7 \\
\hline Púrpura Pigmentar Crônica & 8 & 3,8 \\
\hline Erisipela & 4 & 1.9 \\
\hline Candidíase Plantar & 4 & 1.9 \\
\hline Verruga Plantar & 2 & 0,9 \\
\hline Nenhuma lesão & 19 & 9,0 \\
\hline Amputação & 7 & 3,3 \\
\hline
\end{tabular}

Fonte: Protocolo de pesquisa

A média de lesões dermatológicas por paciente com até cinco anos de doença foi de 1,47 , enquanto que, entre os pacientes com mais tempo de diagnóstico, a média de lesóes foi de 1,8, no entanto, essa diferença náo foi estatisticamente significativa. Analisando os dados, observou-se significante associação entre o tempo de doença e a presença de lesóes dermatológicas, destacando que, quanto maior o tempo de doença, isto é, mais que cinco anos, aumentava a probabilidade de os pacientes terem afecçóes nos pés $(\mathrm{p}=0,01)$.

$\mathrm{Na}$ comparaçáo entre os sexos, observou-se que, entre as mulheres, havia maior proporção de apresentarem pelo menos uma lesão dermatológica quando comparado com os homens $(\mathrm{p}=0,01)$. Além disso, especificamente a xerose foi identificada com maior frequência e estatisticamente significante no gênero feminino $(n=86 ; p=0,03)$. Não houve diferença significativa entre os gêneros com relação à presença das demais afecçóes dermatológicas identificadas no estudo.

Em relaçáo às comorbidades associadas ao curso do DM entre os pacientes envolvidos, a hipertensão arterial sistêmica $(60,8 \%)$ teve lugar de destaque, seguida da dislipidemia $(24,1 \%)$ e da neuropatia periférica $(18,9 \%)$ (Tabela 5).

Tabela 5 - Comorbidades identificadas nos pacientes

continua

\begin{tabular}{l|c|c}
\hline Variáveis & n & $\%$ \\
\hline Comorbidades & 129 & 60,8 \\
\hline HAS* & 51 & 24,1 \\
\hline Dislipidemia & 40 & 18,9 \\
\hline Neuropatia & 19 & 9,0 \\
\hline Tireoide & 13 & 6,1 \\
\hline Retinopatia & 11 & 5,2 \\
\hline Nefropatia & 9 & 4,2 \\
\hline Artrose & 5 & 2,4 \\
\hline Asma & 5 & 2,4 \\
\hline Osteoporose & 2 & 0,9 \\
\hline Hepatite C & &
\end{tabular}


Tabela 5 - Comorbidades identificadas nos pacientes

\begin{tabular}{|c|c|c|}
\hline Variáveis & $\mathbf{n}$ & $\%$ \\
\hline \multicolumn{3}{|c|}{ Comorbidades } \\
\hline Catarata & 2 & 0,9 \\
\hline Cardiopatia & 2 & 0,9 \\
\hline Depressão & 5 & 2,4 \\
\hline Outros & 5 & 2,4 \\
\hline
\end{tabular}

*HAS = Hipertensão Arterial Sistêmica

Fonte: Protocolo de pesquisa

\section{Discussão}

A população do estudo revelou uma predominância do sexo feminino e faixa etária acima de 60 anos (43,9\%). Tais características se mantiveram semelhantes às descritas em outros estudos (BRAGANÇA, 2010; BORTOLETTO; HADDAD; KARINO, 2009; SOUZA, 2008).

Com relação à escolaridade, detectou-se baixo grau de instrução, podendo ser entendida como redutor do acesso a informaçóes sobre a doença e seu controle, favorecendo riscos e desenvolvimento de complicaçóes (SOUZA, 2008). Entende-se que o acesso precário à rede de saúde, a insuficiência de treinamento de profissionais em relação a pé diabético, a fragilidade na regulação de referência e contra referência, bem como registros e monitorização de amputaçôes por DM ineficientes podem agravar a problemática (SBD, 2015).

Dentre os pacientes que declararam ter cuidados com os pés, predominaram aqueles com ensino médio e superior. Esses dados enfatizam a necessidade da implementação de estratégias educacionais e assistenciais acessíveis que facilitem o entendimento e o apoio a essa população, objetivando simplificar a compreensão do autocuidado para uma maior abrangência dos pacientes, independentemente de sua escolaridade (BRAGANÇA, 2010; SOUZA, 2008). Mister ressaltar que as estratégias de educação em saúde devem obrigatoriamente fazer parte do tratamento do DM e se constituem em uma forma de abordar aspectos relevantes para o cuidado com esta doença. Nesse sentido, o alvo da educação em saúde em diabetes é promover o autocuidado e aumentar o conhecimento sobre a doença e, com isso, mudar hábitos que prejudiquem o controle e proporcionem o aparecimento de complicações (AMARAL; TAVARES, 2009).

O nível de escolaridade e a localizaçáo territorial refletem diretamente no conhecimento sobre o DM e suas complicaçóes mais comuns, a forma correta de tratamento e sua adequada prevençáo (SANTOS et al., 2011). Tal averiguaçáo não foi diferente nesta pesquisa. Quando questionados sobre conhecerem a Síndrome do pé diabético, $139(65,6 \%)$ pacientes afirmaram não saber do que se trata. O dado é preocupante, considerando a importância da informação para uma prevenção efetiva desta complicaçáo e que a maioria dos pacientes a desconhece. Deste total, apenas 43 (30,9\%) referiram realizar algum tipo de cuidado com os pés, reafirmando ainda mais a necessidade do conhecimento disseminado.

Os pacientes que já conheciam a Síndrome, em sua maioria, já tinham um cuidado maior com os pés. A baixa escolaridade, em geral, reflete a baixa renda familiar, que também interfere no controle da doença, principalmente em relação à alimentação e aos cuidados de 
higiene e calçados. Esta questão reflete negativamente na saúde do paciente, pois, mesmo que a unidade básica de saúde esteja preparada e equipada com instrumento e pessoal, sem ingesta nutricional adequada e autocuidados básicos a intervenção do profissional de saúde trará benefícios limitados, em virtude dos problemas sociais envolvidos no contexto de vida do paciente (SANTOS et al., 2011).

Quando foi questionada a prática do autocuidado para com o pé diabético, a maioria dos investigados no presente estudo relatou não ter cuidados especiais com os pés, o que de maneira semelhante foi demonstrado no estudo de Souza (2008) e Morais et al. (2009). Estes resultados ressaltam a importância de uma educação para o autocuidado, principalmente no que concerne às lesôes nos pés (MAUES, et al., 2007). O processo educativo deve ser simples, relevante, consistente e contínuo. Está bem estabelecido que $85 \%$ dos problemas decorrentes do pé diabético são passíveis de prevenção, a partir dos cuidados especializados (ROCHA, 2009).

Descrevendo a terapêutica na população estudada, foi observado que 1,9\% estavam realizando tratamento de maneira não farmacológica, apenas com dieta associada à realização de alguma atividade física; 58,5\% faziam uso de hipoglicemiantes orais; 19,8\% associavam a insulina e os hipoglicemiantes orais; e 19,3\% se tratavam apenas com a insulina. Essa diferença na forma de tratamento afirma a prevalência de DM tipo 2 entre os participantes da pesquisa (BORTOLETTO; HADDAD; KARINO, 2009; SOUZA, 2008).

As comorbidades observadas com maior frequência foram a Hipertensão Arterial Sistêmica e a dislipidemia. Esses valores mantiveram a proporcionalidade semelhante a outros estudos na área (THOMAZELLI; MACHADO; DOLÇAN, 2015; FERREIRA; SANTOS, 2009). A presença de vasculopatia foi observada em 11,3\% dos pacientes, valor inferior ao encontrado em outro estudo realizado em um hospital público de Recife (SANTOS et al., 2011), porém não menos importante. A neuropatia periférica esteve presente em $18,9 \%$ do total dos pacientes estudados. Um estudo realizado em Florianópolis determinou que $17,14 \%$ apresentavam sintomas neuropáticos periféricos, e outro realizado em um município de Santa Catarina em 2014, 20,35\% (BRAGA, et al., 2015).

A maioria dos examinados durante o estudo apresentava lesóes cutâneas, resultando uma média de 2 lesóes por pessoa examinada. Outro estudo realizado em 2000, na cidade de Ribeirão Preto, com 403 pacientes, também detectou a presença de alterações cutâneas na maior parte dos pacientes entrevistados, porém com uma média maior que a encontrada em nosso estudo, correspondendo de 3 a 4 lesóes por paciente (3,7\%) (FOSS, 2005). O percentual de pacientes sem nenhuma lesão cutânea também foi superior ao encontrado no presente estudo, $19 \%$ contra $9 \%$.

Este estudo identificou diversos tipos de lesóes dermatológicas, sendo a xerose presente em 55,2\% da população estudada, a onicomicose em 43,9\% e as fissuras em $31,1 \%$, com maior destaque pela sua alta prevalência. Esses valores aproximaram-se dos dados apresentados por outra pesquisa realizada em Ribeirão Preto, onde a xerose foi responsável por $63,4 \%$ das úlceras nos pés dos pacientes diabéticos, a onicomicose por 20,8\%, calos e rachaduras por 49,5\% (SOUZA, 2008; PEDROSA, 2001). A associação de quanto maior o tempo de doença, maior a probabilidade de encontrar lesóes dermatológicas, foi realizada, comparando a população com menos e com mais de cinco anos de diagnóstico de DM, mostrando associação significativa nos resultados deste trabalho $(p=0,01)$.

O comprometimento da pele é fator facilitador para a instalação de um processo infeccioso, podendo atingir planos mais profundos de tecidos subcutâneos, principalmente 
se associados a perdas sensitivas e dolorosas e também a deformidades estruturais, o que pode levar a infecçôes graves e ocasionar a amputação do membro (VIGO, 2006).

\section{Conclusão}

Este estudo demonstrou a elevada frequência de lesôes dermatológicas em pacientes com DM, predominando naqueles com maior tempo de doença e menor grau de escolaridade. Estes achados reforçam a importância de fornecer orientaçóes acessíveis e incentivar o autocuidado dos pacientes, como estratégia de reduzir a ocorrência de complicaçóes nos pés de pacientes diabéticos.

\section{Conflitos de interesse}

Declaramos que não há conflito de interesses na redação do presente artigo.

\section{Referências}

ALAVI, A. et al. Diabetic foot ulcers Part I. Pathophysiology and prevention.Journal of the American Academy of Dermatology, v.70, n. 1, p. 1.e1 - 1. e18, jan. 2014.

ALBERTI, K.G.; ZIMMET, P.Z. Definition, diagnosis and classification of diabetes mellitus and its complications. Part 1: DiagnosisandclassificationofDiabetes Mellitus.Geneva: WHO, 1999.

AMARAL, A.S.; TAVARES, D.M.S. Cuidados com os pés: conhecimento entre pessoas com diabetes mellitus. Revista Eletrônica de Enfermagem, v. 11, n. 4, p. 801-810, 2009.

AMERICAN DIABETES ASSOCIATION. Diagnosis and classification of diabetes mellitus. Diabetes Care, 2013.

BAKKER, K.; APELQVIST, J.; SCHAPER, N.C. Practical guidelines on the management and prevention of the diabetic foot 2011.Diabetes/MetabolismResearchandReviews, v.28, n. 1, p. 225-231, Feb. 2012.

BARBOSA J.H.P.; OLIVEIRA S.L.; SEARA L.T. Produtos da glicação avançada dietéticos e as complicações crônicas do diabetes. Revista de Nutrição, Campinas, v. 22, n. 1, p. 113-124, jan./fev. 2009.

BOADA, A. Lesiones cutâneas em el pie diabético.ActasDermo-Sifiliográficas, v. 103, n. 5, p. 348-356, 2012.

BORTOLETTO, M.S.S.; HADDAD, M.C.L.; KARINO, M.E. Pé diabético, uma avaliação sistematizada. Arq. Ciênc. Saúde Unipar, Umuarama, v. 13, n. 1, p. 37-43, jan./abr. 2009.

BRAGA, D.C. et al. Avaliação de neuropatia e complicações vasculares em pacientes com diabetes mellitus em um município rural de Santa Catarina. Revista da AMRIGS, Porto Alegre, v. 59, n. 2, p. 78-83, abr./jun. 2015.

BRAGANÇA, C.M. et al. Avaliação das práticas preventivas do pé diabético. Journal of the Health Sciences Institute, Campinas, v. 28, n. 2, p.159-163, abr./jul. 2010. 
CAIAFA, J.S. et al. Atenção integral ao portador de Pé Diabético.J. Vasc. Bras., v.10, n. 4, Supl. 2, 2011.

CECILIO, H.P.N. et al. Comportamentos e comorbidades associados as complicações microvasculares do diabetes. Acta Paulista de Enfermagem, São Paulo,v. 28, n. 2, p. 113-119, ago. 2015.

FERREIRA, F.S.; SANTOS, C.B. Qualidade de vida relacionada à saúde de pacientes diabéticos atendidos pela equipe saúde da família. Revista de Enfermagem UERJ, Rio de Janeiro, v. 17, n. 3, p. 406-411, jul./set. 2009.

FOSS, N.T. et al. Dermatoses em pacientes com diabetes mellitus. Revista de Saúde Pública da USP, São Paulo, v. 39, n. 4, p. 677-682, mar. 2005.

LEDESMA, M.S. et al. Complicaciones macrovasculares del paciente diabético. Pie diabético.Medicine, v. 10, n. 17, p. 1110-1124, 2008.

MAUES, C.R. et al. Epidemiologia de idosos internados na enfermaria de clínica médica de hospital público. Rev. Para. Med., Belém, v. 21, n. 3, set. 2007.

MORAIS, G.F.C. et al. Conhecimento e práticas dos diabéticos acerca das medidas preventivas para lesões de membros inferiores. Revista Baiana de Saúde Pública, v. 33, n. 3, p. 361-371, jul./set. 2009.

ROCHA, R.M.; ZANETTI, M.L.; SANTOS, M.A. Comportamento e conhecimento:Fundamentos para prevenção do pé diabético. Acta Paulista de Enfermagem, São Paulo, v. 22, n. 1, p. 17-23, 2009.

SANTOS, I.C.R.V. et al. Amputações por pé diabético e fatores sociais: Implicações para cuidados preventivos de enfermagem. Northeast Network Nursing Journal (Rev.Rene), Fortaleza, v. 12, n. 4, p. 684-691, out./ dez. 2011.

SBD: SOCIEDADE BRASILEIRA DE DIABETES. Atualização Brasileira sobre diabetes. Rio de Janeiro: Diagraphic, 2015.

SBD: SOCIEDADE BRASILEIRA DE DIABETES. Diretrizes da Sociedade Brasileira de Diabetes: 2014-2015. São Paulo: AC Farmacêutica, 2015.

SOUZA M.A. Autocuidado na prevenção de lesões nos pés: conhecimento e prática de pacientes diabéticos.2008. Dissertação (Mestrado) - Universidade Federal da Paraíba, UFPB, João Pessoa, 2008.

THOMAZELLI, F.C.S.; MACHADO, C.B.; DOLÇAN, K.S. Análise do risco de pé diabético em um ambulatório interdisciplinar de diabetes. Revista da AMRIGS, Porto Alegre, v. 59, n. 1, p. 10-14, jan./mar. 2015.

TSCHIEDEL, B. Endocrinologia JBM, v. 102, n. 5, 2014.

VIEIRA-SANTOS, I.C.R. et al. Prevalência de pé diabético e fatores associados nas unidades de saúde da família da cidade do Recife, Pernambuco, Brasil, em 2005. Cadernos de Saúde Pública, Rio de Janeiro, v.24, n.12, p. 2861-2870, dez. 2008.

VIGO, K.O. et al. Caracterização de pessoas com diabetes em unidades de atenção primária e secundária em relação a fatores desencadeantes do pé diabético. Acta Paulista de Enfermagem, São Paulo, v. 19, n. 3, p. 296-303, ago. 2006.

VIGO, K.O; PACE, A.E. Pé diabético: estratégias para prevenção. Acta Paulista de Enfermagem, São Paulo, v. 18, n. 1, p. 100-109, 2005. 